\title{
GIS and GPS based Mapping of Surface and Subsurface Secondary and Micronutrients of Pulse Growing Soils of Ganjam District, Odisha, India
}

\author{
R.K. Nayak ${ }^{1}$, B. Jena ${ }^{1 *}$, J. Das ${ }^{1}$ and A.K. Shukla ${ }^{2}$ \\ Department of Soil Science and Agricultural Chemistry, Micro-Secondary Nutrient \\ Laboratory, OUAT, Bhubaneswar-751003, India \\ *Corresponding author
}

\section{A B S T R A C T}

\section{Keywords}

Mapping, Pulse, Micro-secondary, Surface, Subsurface

Article Info

Accepted:

04 October 2019

Available Online:

10 November 2019
A survey was conducted to map the micro-secondary nutrient status of surface and subsurface soils of pulse growing region of Ganjam to find out the soil related production constraint for pulse growing region of Ganjam district of Odisha where pulses are grown in an area of 20.8 lakh ha with a low production and productivity. For the study GPS based 565 surface samples from 22 blocks of Ganjam district and two pedons (one each from upland and medium land) were collected during dry season, studied in the field and laboratory following standard procedures. The surface soils of Ganjam district were mostly acidic in reaction and non saline. The organic carbon content varied from 0.01 to $1.64 \%$ with 39.82 PSD. The exchangeable calcium and magnesium content varied from 1.08 to $10.80 \mathrm{cmole}\left(\mathrm{p}^{+}\right) / \mathrm{kg}$ and 0.02 to $5.76 \mathrm{cmole}\left(\mathrm{p}^{+}\right) / \mathrm{kg}$. The available sulphur content ranged from 0.25 to $130.60 \mathrm{mg} / \mathrm{kg}$ with $48.08 \mathrm{PSD}$. The DTPA extractable Fe, Mn, Cu ranged from 6.33 to $315.76,2.28$ to 255.76 and 0.02 to $8.32 \mathrm{mg} / \mathrm{kg}$ respectively. The DTPA extractable $\mathrm{Zn}$ content varied from 0.02 to $19.21 \mathrm{mg} / \mathrm{kg}$ with $31.36 \mathrm{PSD}$. The HWS-B content varied from $0.02-11.3 \mathrm{mg} / \mathrm{kg}$ with 52.54 PSD. Multinutrient deficiency of $\mathrm{S}+\mathrm{B}$, $\mathrm{B}+\mathrm{Zn}, \mathrm{S}+\mathrm{Zn}$ and $\mathrm{S}+\mathrm{B}+\mathrm{Zn}$ was $26.55,18.76,18.05$ and 10.97 per cent respectively. GIS based fertility maps for $\mathrm{pH}, \mathrm{S}, \mathrm{Fe}, \mathrm{Mn}, \mathrm{Cu}, \mathrm{Zn}$ and $\mathrm{B}$ were prepared on the basis of standard rating. From the above study for better management and to enhance the production, productivity and quality nutrient rich pulse crops in Ganjam soils application of lime, S, Zn \& B nutrient should be supplemented Proper rates of lime, S, Zn and B should be applied to the pulse growing soils of Ganjam to boost its productivity. Further correlation of soil test data with various soil properties were.

\section{Introduction}

Pulses are important crops, which help in ensuring food and nutritional security by providing protein in the diet. It also helps in maintaining soil health through fixation of atmospheric nitrogen. Pulses are grown in all the thirty districts of Odisha. At present, pulses are grown in 20.8 lakh ha area with production of 10.6 lakh tonnes and productivity of $508 \mathrm{~kg} / \mathrm{ha}$ which far below the national average. With the increase in 
population, consumer awareness and affordability of middle /lower middle and other category citizens up to some degree, the demand of pulses has also increased overtime. Ganjam is the leading district with respect to area and production while Rayagada district has the highest productivity. Ganjam is an agriculturally diversified district with a total geographical area of $8206.00 \mathrm{~km}^{2}$ situated between 19.00' and 20-17' of the North Latitude and 84-6' to 85-11' of East Longitude. It has 22 community development blocks with total population of 35, 20, 151 . The district is under two agro-climatic zones namely East and South eastern coastal plain covering Chatrapur Sub-Divisions and eastern parts of Berhampur and second one is North eastern ghat hill zone covering Bhanjanagar Sub-Division and Western parts of Berhampur. Major cropping systems are paddy-pulses in rainfed red lateritic soils and paddy- green gram in rainfed alluvial soils. But the production and productivity are far below from national average.

Pulses need sulphur, $\mathrm{Ca}, \mathrm{Mg}$ and micronutrients to produce better quality and nutrient rich grains. Hence it is necessary to assess the native nutrient status of the soils of pulse growing region of the state to identify the soil nutrient related production constraint and suggesting remedial measures to enhance the production and productivity of pulses.

\section{Materials and Methods}

For the collection of GPS based soil samples soil sampling grids of one sample $/ 5 \mathrm{~km}^{2}$ areas were pre-determined systematically for each community block from revenue map. Twenty five numbers of surface soil samples collected from each block and sampling was done with the help of GPS (Global positioning system) instrument. Latitude and Longitude recorded for each soil sample. The soil samples were collected from $15 \mathrm{~cm}$ depth (Muhr et al.,
1965) with the help of phaurah. A V-shaped cut made up to the plough layer and a uniform $1.5 \mathrm{~cm}$ thick slice taken out. A total of 550 surface soil samples were collected from 22 blocks covering the different land situations. The collected soil sample thoroughly mixed on a clean piece of polythene sheet, processed and kept it in plastic bottle with suitable description and identification code numbers.

\section{Soil profile exposure and sample collection}

Two Pedons representing up and medium land were studied in Ganjam district. One at Ekalpur Soroda belongs to medium land other one at CPR, Ganjam, Ankushpur belongs to up land soil samples from genetic horizons were collected.

After processing soils were analysed for various physico-chemical properties as well as nutrients. The colour of the soil samples in clod form air dried condition were determined by matching the colour with Munsell soil colour chart (1954). Percentage of sand, silt and clay were determined with the help of the formula (Piper, 1950) and the textural classes were determined by the help of textural triangle (International system).

$\mathrm{pH}$ and EC of the soil samples were determined in Soil: water extraction in 1:2.5 ratio as per the method of Jackson, 1973.

Organic carbon was determined by Walkley and Black (1934) rapid titration method.

Available Sulphur in the soil was determined by turbidometric method (Chesnin and Yien, 1951). Exchangeable $\mathrm{Ca}^{2+}$ plus $\mathrm{Mg}^{2+}$ of soil was determined as per the method outlined in Page and Miller (1982).

Available boron in soil was determined by Hot water soluble method of Berger and $\operatorname{Trug}(1939)$ followed by measurement of 
colour intensity at $420 \mathrm{~nm}$ wave length in Systronics Spectrophotometer model 166(John et al., 1975).

Available micronutrients $\mathrm{Zn}, \mathrm{Cu}, \mathrm{Mn}$ and $\mathrm{Fe}$ in soils were estimated by DTPA extraction method of Lindsay and Novell (1978).

After analysis the data are subjected to descriptive statics and deficiency percent and multinutrient deficiency were computed. Then data are fed to GIS software along with latitude and longitude for development of fertility maps for pulse growing areas of Ganjam,Odisha.

\section{Results and Discussion}

Secondary nutrients are required less than primary nutrients but more than the micronutrients. Deficiency in soils may results deficiency in plants. Hence the soil status of any nutrients is an indicator for availability of that nutrient for plants. Micro nutrients although required small quantity compared to primary and secondary nutrients but no way less important than these nutrients. There role is very crucial in crop production and quality of crop. These also help in utilisation of primary and secondary nutrients in plant metabolism. Therefore deficiency or toxicity of micronutrients leads to not only loss of yield but also quality and affect the use efficiency of primary and secondary nutrients. Surface soil samples from different blocks of Ganjam district were analysed and presented in Tables 1 and 2. The soil reaction $(\mathrm{pH})$ of in surface soil of Ganjam district were varied from 4.66 to 7.93 with $50 \%, 37 \%$ and $13 \%$ were acidic, neutral and alkaline range respectively (Table 3). Upland red lateritic soils were mostly acidic in nature which needs liming for amelioration. The plain medium alluvium were neutral, alkalinity in soil reaction were due to basic parent material and coastal salinity. The EC varied from 0.01 to
0.82 showed normal for plant growth. The places nearer to under the clutch of sea water inundation at present or past showed higher value of EC due to presence of more soluble salts. The organic carbon content varied from 0.01 to $1.64 \%$ with $39.82,28.18,32$ percentage of low, medium and high range respectively. The higher organic carbon content found under coastal low land and nearer to forest area.

Distribution of Secondary nutrients like Exch $\mathrm{Ca}$ and $\mathrm{Mg}$ is presented in Table 4 and that of micronutrients in Table 5 and 6.

\section{Exchangeable calcium}

The exch.Ca value ranges between 1.08 to 10.80 Cmole $(p+) / k g$ with a mean value of 6.74 Cmole $(p+) / \mathrm{kg}$. Maximum value of exchangeable calcium in soil was found in Buguda, Digapahandi, Ganjam, Seragada and Belaguntha block where as minimum value was in Kukudakhandi, Aska, Sanakhemundi blocks.

\section{Exchangeable magnesium}

Exchangeable magnesium content of surface soils varied from 0.62 to 5.76 Cmole $(\mathrm{p}+) / \mathrm{kg}$ with an average value of 2.75 Cmole $(\mathrm{p}+) / \mathrm{kg}$. Among different blocks $20 \%$ samples were deficient in Bhanjanagar block followed by Sanakhemundi block showing 12\% sample deficiency.

\section{Available sulphur}

Available $\mathrm{S}$ is the most limiting secondary nutrient in Indian soils. This type of observation given by Shukla (2013).

From crops requirement angle it stands next to NPK. From the table the Sulphur content varied from 0.25 to $130.6 \mathrm{mg} / \mathrm{kg}$ with an district average of $17.37 \mathrm{mg} . / \mathrm{kg}$. There was 
$48.08 \%$ deficiency of $\mathrm{S}$ in Ganjam soil. Deficiency of $\mathrm{S}$ was found in all blocks with PSD ranging from $20 \%$ in Patrapur block to $72 \%$ in Beguniapada block. Similar results were found by Sahrawath et al., (2007) and Mishra et al., (2016).

\section{Micro nutrients}

The DTPA-Fe, $\mathrm{Mn}, \mathrm{Cu}$, ranged from 6.33 to $315.76 \mathrm{mg} / \mathrm{kg}, 2.28$ to $255.76 \mathrm{mg} / \mathrm{kg}$ and 0.02 to $8.32 \mathrm{mg} / \mathrm{kg}$ respectively and these were found mostly sufficient with deficiency of $0.07 \% \mathrm{Mn}$ and $1 \% \mathrm{Cu}$. The parent materials were rich in these elements (Table 7 and 8).

The DTPA-Zn content varied from 0.02 to $19.21 \mathrm{mg} / \mathrm{kg}$ with $31.36 \%$ samples were deficient. The deficiency of $\mathrm{Zn}$ was mostly observed in medium land and calcareous soil.

For better growth and development zinc fertilization@ @ kg/ ha for rice or @ $2 \mathrm{~kg} / \mathrm{ha}$ for non rice crops are recommended. In acute $\mathrm{Zn}$ deficiency soils farmer can go for soil application and foliar spray b@ $0.5 \% \mathrm{ZnSO}_{4}$ solution.

\section{Hot water soluble boron}

The hot water soluble boron (HWS-B) content in surface soils of Ganjam district varied from 0.02 to $11.30 \mathrm{mg} / \mathrm{kg}$ with a mean value of 1.10 $\mathrm{mg} / \mathrm{kg}$ and $52.54 \%$ samples were found deficient.

The most deficient samples were observed in Rangeilunda, Patrapur Beguniapada and Jagannathprasad blocks. Similar extent of Boron deficiency was reported by Sahrawath et al., (2007), not only in Mahaboobnagar district of Telangana State but also in different states of India.

Digitised soil fertility maps for S, B, Zn, Fe, $\mathrm{Mn}, \mathrm{Cu}$ were prepared with the help of ARC GIS software and presented in figure 1.

\section{Multi nutrient deficiency}

In some surface soil sample not only one but several nutrient deficiency was observed. The multinutrient deficiency in surface soil of district were calculated and found that the extent of percentage deficiency of $\mathrm{S}+\mathrm{B}, \mathrm{B}+\mathrm{Zn}$, $\mathrm{S}+\mathrm{Zn}$ and $\mathrm{S}+\mathrm{B}+\mathrm{Zn}$ was found to be 26.55 , 18.76, 18.05 and 10.97 respectively. Similar type of results was founded by Shukla et al., (2014).

Hence from the analysis result pulse growing soils exhibited highest deficiency of Boron followed by $\mathrm{S}$ and then $\mathrm{Zn}$. Hence pulses like greengram, blackgram, cowpea production may be affected due to their deficiency in soil whereas arhar, gram due to their long root length will be able to meet these deficient nutrients from subsurface soil. The deficiency of boron was due to light texture, acidic nature, non application of boron fertilizer and growing boron loving crops.

The exchangeable calcium and magnesium content in up and medium land soils were sufficient contributed by parent material where as available sulphur was found deficient due to low organic matter content of the soil. The DTPA-Fe, $\mathrm{Mn}, \mathrm{Cu}$ were sufficient in both the pedon with higher value in pedon1 compared to pedon 2 .

But the available $\mathrm{Zn}$ and $\mathrm{B}$ content was more in pedon 2 compared to pedon 1 in respectively horizon. Most of the surface soils are acidic with poor organic carbon without any salt hazard. Sulphur is a limiting secondary nutrient giving an indication of its application through fertilizer to different crops as it is required for pulse crops. $\mathrm{Fe}, \mathrm{Mn}$ and $\mathrm{Cu}$ are sufficient in surface soil where as the $\mathrm{Zn}$ and $\mathrm{B}$ are two micronutrient posing constraints for crop production which must be emphasised for balanced nutrition. 
Table.1 Morphological and physical properties of pedon soils

\begin{tabular}{|c|c|c|c|c|c|c|c|c|c|c|}
\hline \multicolumn{11}{|c|}{ Pedon 1} \\
\hline \multirow{3}{*}{$\begin{array}{c}\text { Genetic } \\
\text { Horizons }\end{array}$} & \multirow{3}{*}{$\begin{array}{c}\text { Depth } \\
\text { (cm) }\end{array}$} & \multirow{3}{*}{ Structure } & & PD & \multirow{3}{*}{$\begin{array}{c}\text { pore } \\
\text { space } \\
(\%)\end{array}$} & \multirow[t]{3}{*}{ Colour } & \multirow{3}{*}{$\begin{array}{c}\text { Sand } \\
(\%)\end{array}$} & \multirow{3}{*}{$\begin{array}{l}\text { Silt } \\
(\%)\end{array}$} & \multirow{3}{*}{$\begin{array}{l}\text { Clay } \\
(\%)\end{array}$} & \multirow[t]{3}{*}{ Texture } \\
\hline & & & סוס & & & & & & & \\
\hline & & & \multicolumn{2}{|c|}{$\mathrm{Mg} / \mathrm{m}^{3}$} & & & & & & \\
\hline Ap & $0-22$ & $\begin{array}{c}\text { Subangula } \\
\text { r blocky }\end{array}$ & $\begin{array}{c}1.4 \\
8\end{array}$ & $\begin{array}{c}2.3 \\
0\end{array}$ & 36 & $\begin{array}{l}\text { Light } \\
\text { grey }\end{array}$ & 80.4 & 8.8 & $\begin{array}{c}10.8 \\
0\end{array}$ & $\begin{array}{l}\text { Loamy } \\
\text { sand }\end{array}$ \\
\hline $\mathbf{B t}_{1}$ & $22-54$ & $\begin{array}{l}\text { Angular } \\
\text { blocky }\end{array}$ & $\begin{array}{c}1.5 \\
6\end{array}$ & $\begin{array}{c}2.3 \\
6\end{array}$ & 34.50 & $\begin{array}{l}\text { Browni } \\
\text { sh } \\
\text { yellow }\end{array}$ & 78.4 & 7.6 & 14 & $\begin{array}{l}\text { Sandy } \\
\text { loam }\end{array}$ \\
\hline $\mathbf{B t}_{2}$ & $\begin{array}{r}54- \\
82+\end{array}$ & $\begin{array}{l}\text { Angular } \\
\text { blocky }\end{array}$ & $\begin{array}{c}1.6 \\
3\end{array}$ & $\begin{array}{c}2.4 \\
8\end{array}$ & 34.20 & $\begin{array}{l}\text { Strong } \\
\text { brown }\end{array}$ & 69.4 & 4.6 & 26 & $\begin{array}{l}\text { Sandy } \\
\text { clay } \\
\text { loam }\end{array}$ \\
\hline \multicolumn{11}{|c|}{ Pedon 2} \\
\hline Ap & $0-16$ & $\begin{array}{c}\text { Subangula } \\
\text { r blocky }\end{array}$ & $\begin{array}{c}1.3 \\
5\end{array}$ & $\begin{array}{c}2.3 \\
0\end{array}$ & 41.30 & $\begin{array}{l}\text { Greyish } \\
\text { brown }\end{array}$ & 67.4 & $\begin{array}{c}21 . \\
6\end{array}$ & 11 & $\begin{array}{l}\text { Sandy } \\
\text { loam }\end{array}$ \\
\hline $\mathrm{Bw}_{1}$ & $18-48$ & $\begin{array}{l}\text { Angular } \\
\text { blocky }\end{array}$ & $\begin{array}{c}1.4 \\
0\end{array}$ & $\begin{array}{c}2.3 \\
2\end{array}$ & 39.65 & $\begin{array}{l}\text { Light } \\
\text { grey }\end{array}$ & 56.6 & $\begin{array}{c}19 . \\
6\end{array}$ & $\begin{array}{c}23.8 \\
0\end{array}$ & loam \\
\hline $\mathrm{Bw}_{2}$ & $\begin{array}{l}48- \\
102\end{array}$ & $\begin{array}{l}\text { Angular } \\
\text { blocky }\end{array}$ & $\begin{array}{c}1.5 \\
8\end{array}$ & $\begin{array}{c}2.4 \\
6\end{array}$ & 35.77 & $\begin{array}{l}\text { Greyish } \\
\text { brown }\end{array}$ & 46.4 & $\begin{array}{c}18 . \\
6\end{array}$ & 35 & $\begin{array}{l}\text { Clay } \\
\text { loam }\end{array}$ \\
\hline
\end{tabular}

Table.2 Secondary-micronutrient content of pedon soils of Ganjam,Odisha

\begin{tabular}{|c|c|c|c|c|c|c|c|c|c|c|}
\hline Pedon & $\begin{array}{c}\text { Genetic } \\
\text { Horizons }\end{array}$ & & $\begin{array}{c}\text { Depth }(\mathbf{c m}) \\
{[\mathbf{c m o l}} \\
(\mathbf{p}+\mathbf{k g}]\end{array}$ & $\begin{array}{c}\mathbf{M g} \\
{[\mathbf{c m o l}}\end{array}$ & $\begin{array}{c}\mathbf{S} \\
(\mathbf{m}+\mathbf{k g}]\end{array}$ & $\begin{array}{c}\mathbf{F e} \\
(\mathbf{m g} / \mathbf{k g})\end{array}$ & $\begin{array}{c}\mathbf{M n} \\
(\mathbf{m g} / \mathbf{k g})\end{array}$ & $\begin{array}{c}\mathbf{C u} \\
(\mathbf{m g} / \mathbf{k g})\end{array}$ & $\mathbf{Z n}(\mathbf{m g} / \mathbf{k g})$ & $\mathbf{B}$ \\
\hline $\mathbf{1}$ & $\mathrm{Ap}$ & $0-12$ & 4.40 & 2.18 & 8.20 & 85.96 & 21.72 & 0.86 & 0.52 & 0.41 \\
\hline & $\mathrm{Bt}_{1}$ & $12-42$ & 4.50 & 2.80 & 6.20 & 73.76 & 8.24 & 0.54 & 0.44 & 0.36 \\
\hline & $\mathrm{Bt}_{2}$ & $42-82$ & 5.60 & 2.98 & 3.10 & 54.56 & 5.60 & 0.33 & 0.36 & 0.10 \\
\hline $\mathbf{2}$ & $\mathrm{Ap}$ & $0-16$ & 14.4 & 4.32 & 11.29 & 30.12 & 12.04 & 0.66 & 0.77 & 0.67 \\
\hline & $\mathrm{Bt}_{1}$ & $18-48$ & 17.88 & 7.70 & 9.97 & 25.04 & 11.16 & 0.54 & 0.31 & 0.54 \\
\hline & $\mathrm{Bt}_{2}$ & $48-102$ & 22.52 & 9.40 & 6.99 & 27.6 & 6.40 & 0.24 & 0.29 & 0.32 \\
\hline
\end{tabular}


Table.3 Basic properties of surface soil

\begin{tabular}{|c|c|c|c|c|c|}
\hline \multirow{2}{*}{ SL } & & \multicolumn{2}{|c|}{$\mathbf{p H}(\mathbf{1}: \mathbf{2 . 5})$} & \multicolumn{2}{c|}{ EC(dS/m) } \\
\cline { 3 - 6 } No. & Name of Block & Range & Mean & Range & Mean \\
\hline $\mathbf{1}$ & Khalikote & $5.08-7.93$ & 6.41 & $0.07-0.66$ & 0.20 \\
\hline $\mathbf{2}$ & Beguniapada & $5.04-6.97$ & 6.13 & $0.03-0.39$ & 0.14 \\
\hline $\mathbf{3}$ & Kabisuryanagar & $5.19-7.79$ & 7.07 & $0.08-0.38$ & 0.15 \\
\hline $\mathbf{4}$ & Polsara & $5.03-7.73$ & 6.36 & $0.04-0.17$ & 0.12 \\
\hline $\mathbf{5}$ & Buguda & $5.48-7.38$ & 6.90 & $0.04-0.17$ & 0.10 \\
\hline $\mathbf{6}$ & Chikiti & $5.10-7.61$ & 6.17 & $0.01-0.23$ & 0.09 \\
\hline $\mathbf{7}$ & Patrapur & $5.49-7.14$ & 6.18 & $0.01-0.67$ & 0.11 \\
\hline $\mathbf{8}$ & Rangeilunda & $5.36-7.75$ & 6.98 & $0.01-0.82$ & 0.19 \\
\hline $\mathbf{9}$ & Digapahandi & $5.06-7.55$ & 6.93 & $0.09-0.63$ & 0.25 \\
\hline $\mathbf{1 0}$ & Sanakhemundi & $5.10-7.47$ & 6.90 & $0.07-0.47$ & 0.17 \\
\hline $\mathbf{1 1}$ & Seragada & $5.88-7.33$ & 6.79 & $0.08-0.82$ & 0.23 \\
\hline $\mathbf{1 2}$ & Aska & $5.54-7.68$ & 6.70 & $0.07-0.42$ & 0.20 \\
\hline $\mathbf{1 3}$ & Hinjilicut & $5.63-7.19$ & 6.73 & $0.03-0.35$ & 0.10 \\
\hline $\mathbf{1 4}$ & Chhatrapur & $5.12-7.43$ & 6.28 & $0.07-0.37$ & 0.21 \\
\hline $\mathbf{1 5}$ & Ganjam & $4.74-7.45$ & 6.07 & $0.10-0.82$ & 0.33 \\
\hline $\mathbf{1 6}$ & Purusottampur & $4.66-7.80$ & 5.98 & $0.05-0.61$ & 0.28 \\
\hline $\mathbf{1 7}$ & Jaganathprasad & $5.30-7.49$ & 6.26 & $0.06-0.63$ & 0.22 \\
\hline $\mathbf{1 8}$ & Belaguntha & $4.99-7.75$ & 6.38 & $0.07-0.42$ & 0.22 \\
\hline $\mathbf{1 9}$ & Bhanjanagar & $4.92-7.20$ & 5.70 & $0.11-0.61$ & 0.19 \\
\hline $\mathbf{2 0}$ & Soroda & $5.02-7.39$ & 6.41 & $0.09-0.32$ & 0.19 \\
\hline $\mathbf{2 1}$ & Dharakote & $5.53-7.93$ & 6.65 & $0.08-0.45$ & 0.16 \\
\hline $\mathbf{2 2}$ & Kukudakhandi & $5.05-7.42$ & 6.55 & $0.01-0.38$ & 0.12 \\
\hline & Ganjam district & $\mathbf{4 . 6 6 - 7 . 9 3}$ & $\mathbf{6 . 4 7}$ & $\mathbf{0 . 0 1 - 0 . 8 2}$ & $\mathbf{0 . 1 8}$ \\
\hline
\end{tabular}


Table.4 Secondary nutrient content of Ganjam district

\begin{tabular}{|c|c|c|c|c|c|c|c|c|}
\hline \multirow[t]{2}{*}{$\begin{array}{l}\text { Name of the } \\
\text { Block }\end{array}$} & \multicolumn{2}{|c|}{$\begin{array}{c}\mathrm{Ca}^{2+} \\
(\operatorname{cmole}(\mathbf{p}+) / \mathrm{kg})\end{array}$} & \multicolumn{3}{|c|}{$\begin{array}{c}\mathrm{Mg}^{2+} \\
\left(\mathrm{cmole}^{2+}(\mathbf{p}+) / \mathrm{kg}\right)\end{array}$} & \multicolumn{3}{|c|}{$\mathrm{S}(\mathrm{mg} / \mathrm{kg})$} \\
\hline & Range & Mean & Range & Mean & PSD & Range & Mean & PSD \\
\hline Khalikote & $1.8-9.0$ & 5.90 & $0.92-3.96$ & 2.66 & 4 & $0.25-78.06$ & 14.8 & 64 \\
\hline Beguniapada & $2.52-10.44$ & 7.12 & $1.10-5.04$ & 1.72 & 0 & $1.98-14.6$ & 7.47 & 72 \\
\hline $\begin{array}{c}\text { Kabisuryanaga } \\
\mathbf{r}\end{array}$ & 2.16-10.08 & 7.20 & $1.36-5.40$ & 2.01 & 0 & $3.5-28.8$ & 8.23 & 68 \\
\hline Polsara & $1.44-9.72$ & 6.98 & $0.72-5.04$ & 2.80 & 8 & $2.5-18.09$ & 9.09 & 64 \\
\hline Buguda & $1.80-10.80$ & 8.28 & $1.36-5.76$ & 2.88 & 0 & $1.98-16.11$ & 7.37 & 76 \\
\hline Chikiti & $1.44-10.44$ & 6.01 & $0.72-5.40$ & 2.88 & 8 & $0.5-111.5$ & 10.37 & 64 \\
\hline Patrapur & $1.80-9.36$ & 6.33 & $1.36-5.40$ & 2.8 & 0 & $4.21-85$ & 19.02 & 28 \\
\hline Rangeilunda & $1.44-8.64$ & 6.12 & $1.8-4.32$ & 3.24 & 0 & $7.43-44.61$ & 21.33 & 24 \\
\hline Digapahandi & $1.80-10.80$ & 6.39 & $1.02-5.04$ & 2.61 & 0 & $4.71-99.37$ & 31.61 & 36 \\
\hline Sanakhemundi & $1.08-7.56$ & 6.48 & $0.72-5.76$ & 3.38 & 12 & $3.22-41.6$ & 20.76 & 28 \\
\hline Seragada & $2.52-10.80$ & 8.56 & $1.36-5.04$ & 3.09 & 0 & $5.20-74.55$ & 24.13 & 40 \\
\hline Aska & $1.08-9.72$ & 5.68 & $0.72-5.76$ & 3.88 & 8 & $5.20-46.59$ & 22.63 & 32 \\
\hline Hinjilicut & $1.44-10.44$ & 6.84 & $1.36-5.40$ & 3.24 & 0 & $1.98-130.6$ & 34.62 & 44 \\
\hline Chhatrapur & $1.8-10.80$ & 5.80 & $1.02-5.40$ & 2.32 & 0 & $3.22-83.51$ & 19.66 & 44 \\
\hline Ganjam & $2.16-10.80$ & 7.11 & $0.72-5.76$ & 4.32 & 8 & $2.23-64.33$ & 29.64 & 32 \\
\hline Purusottampur & $2.52-10.44$ & 7.83 & $1.36-5.40$ & 2.97 & 0 & $2.48-64.33$ & 22.58 & 40 \\
\hline Jaganathprasad & 2.16-10.44 & 7.47 & $1.38-3.96$ & 1.89 & 0 & $7.68-57$ & 21.76 & 28 \\
\hline Belaguntha & $1.44-10.80$ & 8.19 & $1.08-5.04$ & 2.79 & 0 & $3.22-31.97$ & 13.31 & 40 \\
\hline Bhanjanagar & $1.80-8.28$ & 4.95 & $0.62-3.24$ & 0.99 & 20 & $0.74-65.9$ & 9.49 & 72 \\
\hline Soroda & $2.16-7.92$ & 5.22 & $1.08-5.04$ & 2.16 & 0 & $3.22-53.03$ & 14.95 & 36 \\
\hline Dharakote & $1.44-9.72$ & 6.19 & $0.72-5.76$ & 3.24 & 8 & $0.25-88.9$ & 12.98 & 60 \\
\hline Kukudakhandi & $1.08-9.36$ & 7.70 & $1.32-4.68$ & 2.80 & 0 & $3.26-10.66$ & 6.47 & 65 \\
\hline Ganjam district & $1.08-10.80$ & 6.74 & $0.62-5.76$ & 2.75 & 3.45 & $0.25-130.6$ & 17.37 & 48.04 \\
\hline
\end{tabular}


Table.5 DTPA-Fe, $\mathrm{Mn}$ and $\mathrm{Cu}(\mathrm{mg} / \mathrm{kg})$ status of soils of Ganjam district

\begin{tabular}{|c|c|c|c|c|c|c|}
\hline \multirow[t]{2}{*}{ Name of Block } & \multicolumn{2}{|l|}{$\mathbf{F e}$} & \multicolumn{2}{|c|}{ Mn } & \multicolumn{2}{|c|}{$\mathbf{C u}$} \\
\hline & Range & Mean & Range & $\begin{array}{l}\text { Mean } \\
\text { (PSD) }\end{array}$ & Range & $\begin{array}{l}\text { Mean } \\
\text { (PSD) }\end{array}$ \\
\hline Khalikote & $8.4-132.96$ & 45.69 & $2.4-172.72$ & $28.04(4)$ & $1.09-4.04$ & 2.39 \\
\hline Beguniapada & $8.72-135.8$ & 39.05 & $18.88-80.76$ & 36.52 & $0.64-2.99$ & 1.91 \\
\hline Kabisuryanagar & $7.44-122.72$ & 38.89 & $8-125.92$ & 29.43 & $0.23-2.89$ & $1.58(4)$ \\
\hline Polsara & $6.32-115.6$ & 39.79 & $8.04-94.76$ & 34.64 & $0.02-5.99$ & $2.19(12)$ \\
\hline Buguda & $9.16-315.76$ & 108.08 & $4-54.76$ & 24.63 & $0.29-2.44$ & $1.08(4)$ \\
\hline Chikiti & $11.44-24.66$ & 116.79 & $6.24-41.24$ & 19.33 & $0.57-3.57$ & 1.69 \\
\hline Patrapur & $20.64-183.52$ & 106.19 & $15.36-93.36$ & 48.56 & $0.92-5.71$ & 2.57 \\
\hline Rangeilunda & $10.52-165.44$ & 61.85 & $2.76-43$ & $17.27(8)$ & $0.35-4.14$ & $1.8(4)$ \\
\hline Digapahandi & $11.6-226.86$ & 86.01 & $12.96-64.68$ & 28.51 & $0.87-5.11$ & 3.6 \\
\hline Sanakhemundi & $6.84-186.36$ & 70.34 & $7.48-104.12$ & 36.44 & $1.37-4.4$ & 3.39 \\
\hline Seragada & 10.24-195.08 & 81.28 & $18.2-101.32$ & 58.82 & $0.65-5.96$ & 3.52 \\
\hline Aska & $14.44-239.12$ & 77.99 & $27.12-131.92$ & 70.57 & $1.63-7.59$ & 4.14 \\
\hline Hinjilicut & $14.4-190.56$ & 81.8 & $10.76-106.72$ & 51.76 & $1.11-4.76$ & 3.52 \\
\hline Chhatrapur & $10.40-214.96$ & 103.55 & $70.96-111.32$ & 44.93 & $0.29-7.56$ & 3.12 \\
\hline Ganjam & $10.40-216.68$ & 104.19 & $2.28-122$ & $50.2(4)$ & $0.54-8.32$ & 3.51 \\
\hline Purusottampur & $12.80-234.88$ & 118.43 & $8.64-122$ & 65.09 & $0.40-8.32$ & 2.99 \\
\hline Jaganathprasad & $27.52-223.76$ & 94.04 & $13.64-167.56$ & 75.68 & $0.89-6.15$ & 2.98 \\
\hline Belaguntha & $46.08-197.72$ & 120.63 & $17.36-105.2$ & 58.36 & $1.06-7.87$ & 4.06 \\
\hline Bhanjanagar & $19.6-252.96$ & 149.73 & $32.88-136.44$ & 88.1 & $0.81-4.39$ & 3.0 \\
\hline Soroda & $9.2-224.24$ & 92.85 & $11.84-255.76$ & 112.32 & $0.83-6.08$ & 3.67 \\
\hline Dharakote & $15.64-206.72$ & 85.03 & $14.08-121.80$ & 48.26 & $0.63-4.70$ & 2.46 \\
\hline Kukudakhandi & $21.6-177.78$ & 79.88 & $6-119.56$ & 43.29 & $0.44-5.04$ & 2.9 \\
\hline Ganjam district & $6.32-315.76$ & 86.71 & 2.28-255.76 & 48.67 & $0.02-8.32$ & 2.82 \\
\hline
\end{tabular}


Table.6 DTPA-Zn and HWS B status of soils of Ganjam district

\begin{tabular}{|c|c|c|c|c|c|c|c|}
\hline \multirow{2}{*}{$\begin{array}{c}\text { Sl. } \\
\text { No. }\end{array}$} & Name of Block & \multicolumn{3}{|c|}{ Zn (mg/kg) } & \multicolumn{3}{c|}{ B (mg/kg) } \\
\hline & & Range & Mean & PSD & Range & Mean & PSD \\
\hline $\mathbf{1}$ & Khalikote & $0.34-2.91$ & 1.06 & 12 & $0.06-7.78$ & 3.1 & 32 \\
\hline $\mathbf{2}$ & Beguniapada & $2.22-8.32$ & 1.29 & 32 & $0.04-9.31$ & 0.72 & 76 \\
\hline $\mathbf{3}$ & Kabisuryanagar & $0.31-1.95$ & 0.71 & 48 & $0.02-11.3$ & 1.72 & 56 \\
\hline $\mathbf{4}$ & Polsara & $0.09-3.03$ & 1.25 & 32 & $0.06-6.15$ & 0.74 & 72 \\
\hline $\mathbf{5}$ & Buguda & $0.02-1.10$ & 0.4 & 48 & $0.02-2.17$ & 0.49 & 64 \\
\hline $\mathbf{6}$ & Chikiti & $0.14-2.36$ & 0.77 & 56 & $0.06-1.91$ & 0.48 & 72 \\
\hline $\mathbf{7}$ & Patrapur & $0.29-9.85$ & 1.61 & 20 & $0.06-0.52$ & 0.28 & 80 \\
\hline $\mathbf{8}$ & Rangeilunda & $0.18-2.50$ & 1.04 & 32 & $0.02-0.76$ & 0.31 & 84 \\
\hline $\mathbf{9}$ & Digapahandi & $0.10-2.41$ & 0.84 & 28 & $0.35-6.52$ & 4 & 20 \\
\hline $\mathbf{1 0}$ & Sanakhemundi & $0.06-8.48$ & 1.4 & 44 & $0.24-5.63$ & 3.43 & 20 \\
\hline $\mathbf{1 1}$ & Seragada & $0.30-2.33$ & 1.14 & 24 & $0.15-2.04$ & 0.71 & 44 \\
\hline $\mathbf{1 2}$ & Aska & $0.34-1.80$ & 1.1 & 24 & $0.13-2.77$ & 1.26 & 28 \\
\hline $\mathbf{1 3}$ & Hinjilicut & $0.23-9.97$ & 1.51 & 28 & $0.04-3.42$ & 0.85 & 48 \\
\hline $\mathbf{1 4}$ & Chhatrapur & $0.16-10.31$ & 2.1 & 8 & $0.15-6.74$ & 0.91 & 48 \\
\hline $\mathbf{1 5}$ & Ganjam & $0.03-9.48$ & 3.03 & 12 & $0.11-1.58$ & 0.76 & 36 \\
\hline $\mathbf{1 6}$ & Purusottampur & $0.03-10.08$ & 2.62 & 24 & $0.17-8.71$ & 1.25 & 40 \\
\hline $\mathbf{1 7}$ & Jaganathprasad & $0.12-4.64$ & 0.99 & 36 & $0.02-0.71$ & 0.33 & 76 \\
\hline $\mathbf{1 8}$ & Belaguntha & $0.24-19.21$ & 2.15 & 28 & $0.24-1.02$ & 0.56 & 52 \\
\hline $\mathbf{1 9}$ & Bhanjanagar & $0.29-13.31$ & 1.72 & 24 & $0.06-1.06$ & 0.56 & 48 \\
\hline $\mathbf{2 0}$ & Soroda & $0.18-2.0$ & 0.85 & 32 & $0.04-1.86$ & 0.6 & 44 \\
\hline $\mathbf{2 1}$ & Dharakote & $0.23-2.07$ & 0.59 & 68 & $0.04-3.73$ & 0.82 & 44 \\
\hline $\mathbf{2 2}$ & Kukudakhandi & $0.30-4.18$ & 0.86 & 30.0 & $0.04-1.41$ & 0.42 & 72 \\
\hline & Ganjam district & $\mathbf{0 . 0 2 - 1 9 . 2 1}$ & $\mathbf{1 . 3 2}$ & $\mathbf{3 1 . 3 6}$ & $\mathbf{0 . 0 2 - 1 1 . 3}$ & $\mathbf{1 . 1 0}$ & $\mathbf{5 2 . 5 4}$ \\
\hline & & & & & & & \\
\hline & & & & & \\
\hline
\end{tabular}


Table.7 DTPA-Fe, $\mathrm{Mn}$ and $\mathrm{Cu}(\mathrm{mg} / \mathrm{kg})$ status of soils of Ganjam district

\begin{tabular}{|c|c|c|c|c|c|c|}
\hline Name of Block & \multicolumn{2}{|c|}{ Fe } & \multicolumn{2}{c|}{ Mn } & \multicolumn{2}{c|}{ Cu } \\
\cline { 2 - 7 } & Range & Mean & Range & $\begin{array}{c}\text { Mean } \\
\text { (PSD) }\end{array}$ & Range & $\begin{array}{c}\text { Mean } \\
\text { (PSD) }\end{array}$ \\
\hline Khalikote & $8.4-132.96$ & 45.69 & $2.4-172.72$ & $28.04(4)$ & $1.09-4.04$ & 2.39 \\
\hline Beguniapada & $8.72-135.8$ & 39.05 & $18.88-80.76$ & 36.52 & $0.64-2.99$ & 1.91 \\
\hline Kabisuryanagar & $7.44-122.72$ & 38.89 & $8-125.92$ & 29.43 & $0.23-2.89$ & $1.58(4)$ \\
\hline Polsara & $6.32-115.6$ & 39.79 & $8.04-94.76$ & 34.64 & $0.02-5.99$ & $2.19(12)$ \\
\hline Buguda & $9.16-315.76$ & 108.08 & $4-54.76$ & 24.63 & $0.29-2.44$ & $1.08(4)$ \\
\hline Chikiti & $11.44-24.66$ & 116.79 & $6.24-41.24$ & 19.33 & $0.57-3.57$ & 1.69 \\
\hline Patrapur & $20.64-183.52$ & 106.19 & $15.36-93.36$ & 48.56 & $0.92-5.71$ & 2.57 \\
\hline Rangeilunda & $10.52-165.44$ & 61.85 & $2.76-43$ & $17.27(8)$ & $0.35-4.14$ & $1.8(4)$ \\
\hline Digapahandi & $11.6-226.86$ & 86.01 & $12.96-64.68$ & 28.51 & $0.87-5.11$ & 3.6 \\
\hline Sanakhemundi & $6.84-186.36$ & 70.34 & $7.48-104.12$ & 36.44 & $1.37-4.4$ & 3.39 \\
\hline Seragada & $10.24-195.08$ & 81.28 & $18.2-101.32$ & 58.82 & $0.65-5.96$ & 3.52 \\
\hline Aska & $14.44-239.12$ & 77.99 & $27.12-131.92$ & 70.57 & $1.63-7.59$ & 4.14 \\
\hline Hinjilicut & $14.4-190.56$ & 81.8 & $10.76-106.72$ & 51.76 & $1.11-4.76$ & 3.52 \\
\hline Chhatrapur & $10.40-214.96$ & 103.55 & $70.96-111.32$ & 44.93 & $0.29-7.56$ & 3.12 \\
\hline Ganjam & $10.40-216.68$ & 104.19 & $2.28-122$ & $50.2(4)$ & $0.54-8.32$ & 3.51 \\
\hline Purusottampur & $12.80-234.88$ & 118.43 & $8.64-122$ & 65.09 & $0.40-8.32$ & 2.99 \\
\hline Jaganathprasad & $27.52-223.76$ & 94.04 & $13.64-167.56$ & 75.68 & $0.89-6.15$ & 2.98 \\
\hline Belaguntha & $46.08-197.72$ & 120.63 & $17.36-105.2$ & 58.36 & $1.06-7.87$ & 4.06 \\
\hline Bhanjanagar & $19.6-252.96$ & 149.73 & $32.88-136.44$ & 88.1 & $0.81-4.39$ & 3 \\
\hline Soroda & $9.2-224.24$ & 92.85 & $11.84-255.76$ & 112.32 & $0.83-6.08$ & 3.67 \\
\hline Dharakote & $15.64-206.72$ & 85.03 & $14.08-121.80$ & 48.26 & $0.63-4.70$ & 2.46 \\
\hline Kukudakhandi & $21.6-177.78$ & 79.88 & $6-119.56$ & 43.29 & $0.44-5.04$ & 2.9 \\
\hline Ganjam district & $\mathbf{6 . 3 2 - 3 1 5 . 7 6}$ & $\mathbf{8 6 . 7 1}$ & $\mathbf{2 . 2 8 - 2 5 5 . 7 6}$ & $\mathbf{4 8 . 6 7}$ & $\mathbf{0 . 0 2 - 8 . 3 2}$ & $\mathbf{2 . 8 2}$ \\
\hline & & & & & & \\
\hline
\end{tabular}

Table.8 Correlation micro-secondary nutrients with soil properties

\begin{tabular}{|c|c|c|c|c|c|c|c|c|c|}
\hline & $\mathbf{p H}$ & $\mathbf{E C}$ & $\mathbf{O C}$ & $\mathbf{B}$ & $\mathbf{S}$ & $\mathbf{F e}$ & $\mathbf{M n}$ & $\mathbf{C u}$ & $\mathbf{Z n}$ \\
\hline $\mathbf{p H}$ & 1.000 & & & & & & & & \\
\hline $\mathbf{E C}$ & $0.204^{* *}$ & 1.000 & & & & & & & \\
\hline $\mathbf{O C}$ & -0.018 & 0.026 & 1.000 & & & & & & \\
\hline $\mathbf{B}$ & 0.073 & 0.058 & -0.089 & 1.000 & & & & & \\
\hline $\mathbf{S}$ & 0.087 & $0.251^{* *}$ & $0.103^{*}$ & 0.011 & 1.000 & & & & \\
\hline $\mathbf{F e}$ & -0.392 & 0.025 & 0.137 & $-0.112^{*}$ & 0.035 & 1.000 & & & \\
\hline $\mathbf{M n}$ & -0.270 & 0.093 & 0.125 & -0.083 & 0.019 & $0.272^{* *}$ & 1.000 & & \\
\hline $\mathbf{C u}$ & -0.123 & 0.176 & $0.101^{*}$ & 0.022 & $0.164^{* *}$ & $0.295^{* *}$ & $0.338^{* *}$ & 1.000 & \\
\hline $\mathbf{Z n}$ & -0.126 & 0.096 & $0.086^{*}$ & 0.023 & $0.122^{*}$ & $0.154^{* *}$ & 0.049 & $0.269^{* *}$ & 1.000 \\
\hline
\end{tabular}


Fig.1 Digitised soil fertility map for Micro-secondary nutrients of Pulse growing soils of Ganjam

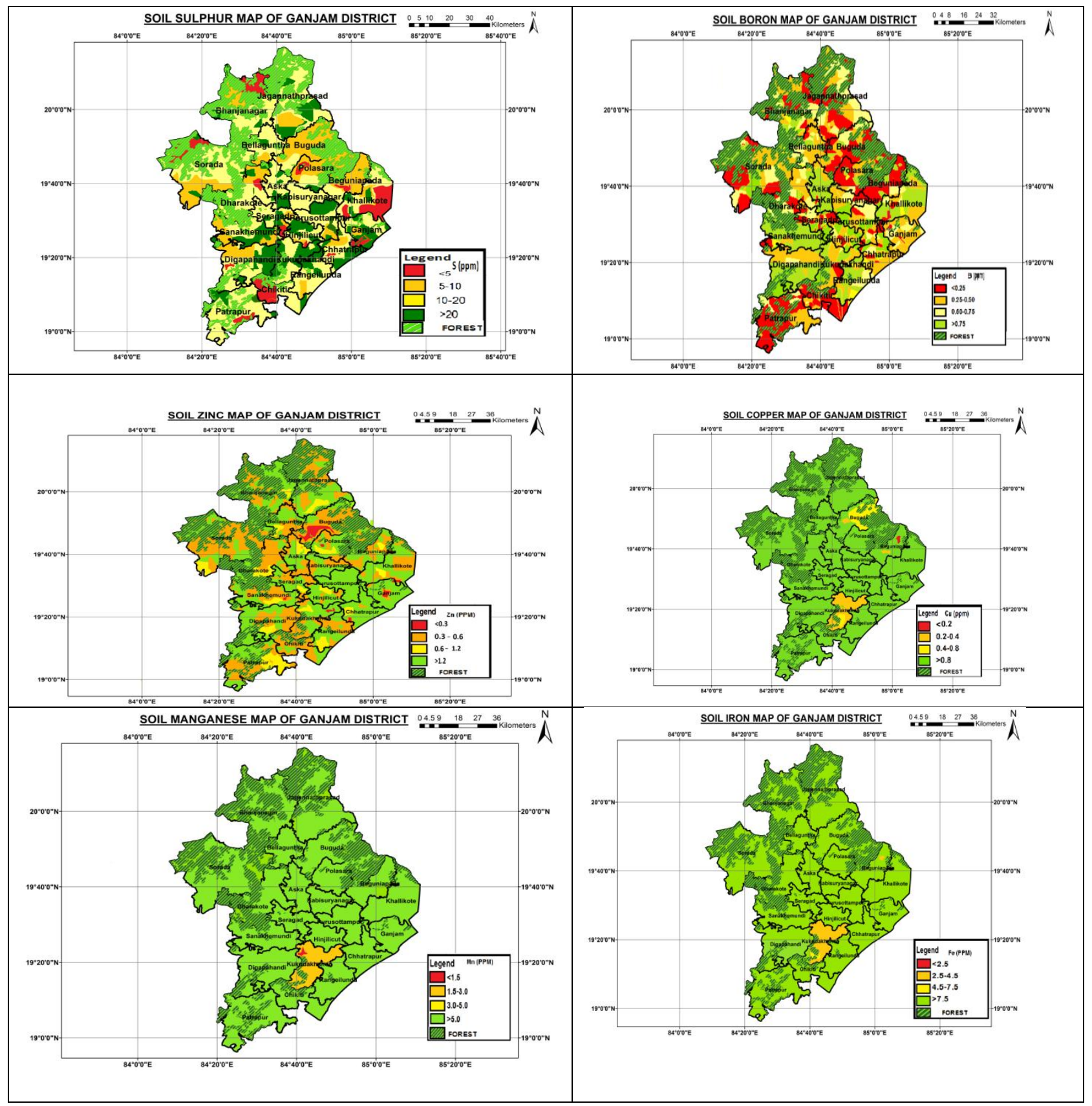


Organic Carbon content of the soil was determined by Wet digestion procedure of Walkley and Black method as outlined in soil chemical analysis (Jackson, 1973). Available Sulphur in the soil was determined by turbidometric method (Chesnin and Yien, 1951).

\section{References}

Berger, K.C. and Truog E. (1939). Boron determination in soil and plants. Indian Eng. Chem. Anal. Ed, 11: 540-545.

Chesnin, L and Yien, C.H.(1951). Turbidimetric determination of available sulphur. Soil Science Society of America Proceedings, 15: 149-151.

Muhr, G.R., Datta, N.P., SankaraSubraney, N; Dever F, Lecy, V. K and Donahue R.R. (1965). Soil testing in India. USAID. Mission to India, New Delhi.

Piper, C.S. (1950). Soil and Plant analysis. InterScience Publication., New York.

Jackson ML. (1973). Soil Chemical Analysis. Prentice Hall of India Pvt. Ltd., New Delhi.

John, M.K., Chuah, H. H and Neufeld,J H. (1975). Application of improved azomethine- $\mathrm{H}$ method to the determination of boron in soils and plants, Analytical Letters, 8: 550-68.

Lindsay, W. L and Norvell, W.A. (1978). Development of DTPA soil test for zinc, iron, manganese and copper. Soil Science. Society of America Journal. 42: 421-428.

Page, A.L., Miller, R.H. and Keeney, D.R. (1982). Methods of soil analysis II. Chemical and microbiological properties. $2^{\text {nd }}$ Edition. ASA-SSSA, Madison, USA.

Walkey, A and Black, C.A. (1934). An examination of the degtjareff method for determining the soil organic matter and a proposed modification of the chromic acid titration method, Soil Science. 37, 29-38.

Shukla, A.K., Tiwari, P.K. and Prakash, C (2014). Micronutrients Deficiencies vis-a-vis Food and Nutritional Security of India, Indian J. Fert., 10 (12), pp.94-112

Munsell Soil colour chart,(1954) Munsell colour company, Batrimore, marieland, 21218, USA.

Sahrawath, K.L, Wani, S.P, Rego, T.J, Parthasarathi $G$ and Murthy, KVS. (2007). Widespread deficiency of S, B, $\mathrm{Zn}$ in dryland soils of the Indian semi arid tropics. Current science I vol93. No 10.

Mishra, A.; Das, D; Saren, S and Dey, P. (2016).GPS and GIS based soil fertility maps of Nayagarh district, Odisha annals of plant and soil research 18(1): 23-28.

\section{How to cite this article:}

Nayak, R.K., B. Jena, J. Das and Shukla, A.K. 2019. GIS and GPS based Mapping of Surface and Subsurface Secondary and Micronutrients of Pulse Growing Soils of Ganjam District, Odisha, India. Int.J.Curr.Microbiol.App.Sci. 8(11): 122-133.

doi: https://doi.org/10.20546/ijcmas.2019.811.015 